\title{
Relative Equilibria of a Satellite Moving in an Elliptic Orbit Subjected to Gravitational and Aerodynamic Torques
}

\author{
Kumari Ranjana \\ Department of Mathematics, Lakshmibai College, University of Delhi, New Delhi, India \\ Email: ranjana2710@yahoo.co.in
}

Received 12 February 2014; revised 6 March 2014; accepted 13 March 2014

Copyright (C 2014 by author and Scientific Research Publishing Inc.

This work is licensed under the Creative Commons Attribution International License (CC BY). http://creativecommons.org/licenses/by/4.0/

(c) (i) Open Access

\begin{abstract}
Here we consider the attitude motion of a satellite, subjected to gravitational and aerodynamic torques in an elliptic orbit. The determination of orientation of equilibrium points has been discussed. It is found that they coincide with those for the circular case as studied by Sarychev and others in the works [1]-[3] and besides we have aimed at the sufficient condition for stability in the sense of Lyapunov.
\end{abstract}

\section{Keywords}

Satellite; Gravitational and Aerodynamic Torques; Equilibria; Condition of Stability

\section{Introduction}

As investigated by [1], it has been found that in the central Newtonian force field a satellite with different moments of inertia has stable equilibrium orientations in a circular orbit. An extensive analysis made by [2] also confirmed the results and he also derived sufficient conditions for stability. Realizing the importance of aerodynamic drag effect on the altitude motion of a satellite in high altitudes, the study was first taken up by [3] restricted to circular orbit taking into consideration the combined effect of gravitational and aerodynamic torques. [1] examined how aerodynamic torque influence on equilibria and dynamics of two rigid bodies connected by spherical hinge. Later an interesting investigation was performed by [4] and they showed that aerodynamic torque can have small none conservative components resulting in instability of the satellite's equilibria. Sazonov obtained the most profound results concerning the loss of satiability of a satellite subjected to aerodynamic torque. The basic problems of the satellite's dynamics with an aerodynamic attitude central system have been discussed by many authors, namely, Sarychev [5]-[7] and Dranovsky. 
As detailed by [2], various forces influence the attitude motion of a satellite and among them we may mention circular radiation effect, atmospheric drag, relativistic effect and many others. But the effect of the atmospheric drag and the later forces can be neglected and so we have mainly concentrated on aerodynamic and gravitational force.

In the present investigation, different from the earlier studies made, we have taken the orbit of the satellite to be elliptic. Although in the first instance the system reduces to a non-autonomous system, since the angular velocity factor cancels out, the variation in the orbit does not matter. However, since the generalized integral of energy reduces to a quadrature direct application of Lyapunov theorem for stability is not applicable and it is now as an open problem to find the condition of stability in the reduced non-autonomous system.

\section{Equations of Motion}

To write the equations of motion we introduce two right-handed Cartesian coordinate system [5] with the origin at the satellite's center of mass $\mathrm{O}$. Oxyz is the orbital reference frame. The axis OZ is directed along the radius vector from the earth center of mass to the satellite one, the axis OX is on the orbit plane \& in direction of the satellites orbital motion. OXYZ is the satellites triad reference frame: $O_{x}, O_{y}, O_{z}$ are principal axes of inertia of the satellite.

Using the body 2-3-1 sequence of angles $\alpha, \beta, \gamma$, we get the following relations between the systems $O X Y Z$ and oxyz

$$
\left[\begin{array}{l}
X \\
Y \\
Z
\end{array}\right]=\left[\begin{array}{ccc}
\cos \alpha & 0 & \sin \alpha \\
0 & 1 & 0 \\
-\sin \alpha & 0 & \cos \alpha
\end{array}\right]\left[\begin{array}{ccc}
\cos \beta & -\sin \beta & 0 \\
\sin \beta & \cos \beta & 0 \\
0 & 0 & 1
\end{array}\right]\left[\begin{array}{ccc}
1 & 0 & 0 \\
0 & \cos \gamma & -\sin \gamma \\
0 & \sin \gamma & \cos \gamma
\end{array}\right]\left[\begin{array}{l}
x \\
y \\
z
\end{array}\right]
$$

Finally the direction cosines of the axes $O_{x}, O_{y}, O_{z}$ in the reference frame $O X Y Z$ are written as

$$
\begin{aligned}
& \cos (x, X)=a_{11}=\cos \alpha \cos \beta \\
& \cos (y, X)=a_{12}=\sin \alpha \sin \gamma-\cos \alpha \sin \beta \cos \gamma \\
& \cos (z, X)=a_{13}=\sin \alpha \cos \gamma+\cos \alpha \sin \beta \sin \gamma \\
& \cos (x, Y)=a_{21}=\sin \beta \\
& \cos (y, Y)=a_{22}=\cos \beta \cos \gamma \\
& \cos (z, Y)=a_{23}=-\cos \beta \sin \gamma \\
& \cos (x, Z)=a_{31}=-\sin \alpha \cos \beta \\
& \cos (y, Z)=a_{32}=\cos \alpha \sin \gamma+\sin \alpha \sin \beta \cos \gamma \\
& \cos (z, Z)=a_{33}=\cos \alpha \cos \gamma-\sin \alpha \sin \beta \sin \gamma
\end{aligned}
$$

We shall let the satellite be subjected to aerodynamic and gravitational torques as in the work [5] and avoiding the repetition, we shall use the same notations as used in the referred paper and the equations of motion subjected to the referred torques as follows:

$$
\begin{gathered}
A \dot{p}+(C-B) q r=3 \omega^{2}(C-B) a_{32} a_{33}+\omega^{2}\left(h_{2} a_{13}-h_{3} a_{12}\right) \\
B \dot{q}+(A-C) r p=3 \omega^{2}(A-C) a_{31} a_{33}+\omega^{2}\left(h_{3} a_{11}-h_{1} a_{13}\right) \\
C \dot{r}+(B-A) p q=3 \omega^{2}(B-A) a_{31} a_{32}+\omega^{2}\left(h_{1} a_{12}-h_{2} a_{11}\right) \\
p=(\dot{\alpha}+\omega) a_{21}+\dot{\gamma}=\bar{p}+\omega a_{21} \\
q=(\dot{\alpha}+\omega) a_{22}+\dot{\beta} \arcsin \gamma=\bar{q}+\omega a_{22} \\
r=(\dot{\alpha}+\omega) a_{23}+\dot{\beta} \cos \gamma=\bar{r}+\omega a_{23} \\
h_{1}=-\frac{Q a}{\omega^{2}}, h_{2}=-\frac{Q b}{\omega^{2}}, h_{3}=-\frac{Q c}{\omega^{2}}
\end{gathered}
$$




$$
\omega=\dot{\theta}=\omega_{0}(1+e \cos \theta)^{2}
$$

$\theta=$ the line anomaly of the elliptic orbit,

$e=$ the eccentricity of the elliptic orbit,

$$
r=\frac{p}{1+e \cos \theta}, \quad \omega_{0}=\frac{\mu}{p}, p=a\left(1-e^{2}\right), \quad a_{12} a_{23}-a_{13} a_{22}=a_{31}
$$

And similar other relations

$$
\begin{aligned}
& \dot{a}_{31}=r a_{32}-q a_{33}+\omega a_{11} \\
& \dot{a}_{32}=p a_{33}-r a_{31}+\omega a_{12} \\
& \dot{a}_{33}=q a_{31}-p a_{32}+\omega a_{13} \\
& \dot{a}_{11}=r a_{12}-q a_{13}-\omega a_{31} \\
& \dot{a}_{12}=p a_{13}-r a_{11}-\omega a_{32} \\
& \dot{a}_{13}=q a_{11}-p a_{12}-\omega a_{33} \\
& \dot{a}_{21}=r a_{22}-q a_{23} \\
& \dot{a}_{22}=p a_{23}-r a_{21} \\
& \dot{a}_{23}=q a_{21}-p a_{22} \\
& (.)=\frac{\mathrm{d}}{\mathrm{d} t}
\end{aligned}
$$

Now (2)p $+(3) q+(4) r$ will give

$$
\begin{aligned}
& A p \dot{p}+B q \dot{q}+C r \dot{r} \\
&=3 \omega^{2}\left[\left(q a_{33}-r a_{31}\right) A_{31}+\left(r a_{31}-p a_{33}\right) B a_{32}+\left(p a_{32}-q a_{31}\right) C a_{33}\right] \\
&\left.+\omega^{2}\left[h_{1}\left(a_{12} r-a_{13} q\right)+h_{2}\left(a_{13} p-a_{11} r\right)\right)+h_{3}\left(a_{11} q-a_{12} p\right)\right]
\end{aligned}
$$

Using the relations (9) and (10), we may write (13) as

$$
\begin{aligned}
& A p \dot{p}+B q \dot{q}+C r \dot{r} \\
&=3 \omega^{2}\left[\left(-\dot{a}_{31}-\omega a_{11}\right) A a_{31}++\left(-\dot{a}_{32}-\omega a_{12}\right) B a_{32}+\left(-\dot{a}_{33}+\omega a_{13}\right) C a_{33}\right] \\
&+\omega^{2}\left[h_{1}\left(\dot{a}_{11}+\omega a_{31}\right)+h_{2}\left(\dot{a}_{12}+\omega a_{32}\right)+h_{3}\left(\dot{a}_{13}+a_{33}\right)\right] \\
& \Rightarrow \frac{1}{2} \frac{\mathrm{d}}{\mathrm{d} t}\left(A p^{2}+B q^{2}+C r^{2}\right)+\frac{3 \omega^{2}}{2} \frac{\mathrm{d}}{\mathrm{d} t}\left(A a_{31}^{2}+B a_{32}^{2}+C a_{33}^{2}\right) \\
&-\omega^{2}\left(h_{1} a_{11}+h_{2} a_{12}+h_{3} a_{13}\right) \\
&=3 \omega^{2}\left(A a_{11} a_{31}+B a_{12} a_{32}+C a_{13} a_{33}\right)+\omega^{3}\left(h_{1} a_{31}+h_{2} a_{32}+h_{3} a_{33}\right)
\end{aligned}
$$

Next (2) $\omega a_{21}+(3) \omega a_{22}+(4) a_{23}$ will give

$$
\begin{aligned}
\omega & {\left[A \dot{p} a_{21}+B \dot{q} a_{22}+C \dot{r} a_{23}\right]+\omega\left[A p\left(r a_{22}-q a_{23}\right)+B q\left(p a_{23}-r a_{21}\right)+C r\left(q a_{21}-p a_{22}\right)\right] } \\
= & 3 \omega^{3}\left[A a_{31}\left(a_{22} a_{33}-a_{32} a_{23}\right)+B a_{32}\left(a_{31} a_{23}-a_{32} a_{21}\right)+C a_{33}\left(a_{32} a_{21}-a_{31} a_{22}\right)\right] \\
& +\omega^{3}\left[h_{1}\left(a_{12} a_{23}-a_{13} a_{22}\right)+h_{2}\left(a_{13} a_{21}-a_{11} a_{23}\right)+h_{3}\left(a_{11} a_{22}-a_{12} a_{21}\right)\right] \\
\Rightarrow & \omega\left[\left(\dot{p} a_{21}+p \dot{a}_{21}\right)+\left(\dot{q} a_{22}+q \dot{a}_{22}\right)+\left(\dot{r} a_{23}+r \dot{a}_{23}\right)\right] \\
& =3 \omega^{3}\left(A_{31} a_{11}+B a_{32} a_{12}+C a_{33} a_{13}\right)+\omega^{3}\left(h_{1} a_{31}+h_{2} a_{32}+h_{3} a_{33}\right)
\end{aligned}
$$

Hence (14)-(15) will give 


$$
\begin{aligned}
& \frac{1}{2} \frac{\mathrm{d}}{\mathrm{d} t}\left(A p^{2}+B q^{2}+C r^{2}\right)-\omega \frac{\mathrm{d}}{\mathrm{d} t}\left(A p a_{21}+B q a_{22}+C r a_{23}\right) \\
& +\frac{3 \omega^{2}}{2} \frac{\mathrm{d}}{\mathrm{d} t}\left(A a_{31}^{2}+B a_{32}^{2}+C a_{33}^{2}\right)-\omega^{2} \frac{\mathrm{d}}{\mathrm{d} t}\left(h_{1} a_{11}+h_{2} a_{12}+h_{3} a_{13}\right)=0
\end{aligned}
$$

Since $\omega$ is a function of $t$, then integration will result to

$$
\begin{aligned}
& \frac{1}{2}\left(A p^{2}+B q^{2}+C r^{2}\right)-\omega\left(A p a_{21}+B q a_{22}+C r a_{23}\right) \\
& +\frac{3 \omega^{2}}{2}\left(A a_{31}^{2}+B a_{32}^{2}+C a_{33}^{2}\right)-\omega^{2}\left(h_{1} a_{31}+h_{2} a_{32}+h_{3} a_{33}\right) \\
& =-\int\left(A p a_{21}+B q a_{22}+C r a_{23}\right) \mathrm{d} \omega+3 \int\left(A a_{31}^{2}+B a_{32}^{2}+C a_{33}^{2}\right) \omega \mathrm{d} \omega \\
& \quad-2 \int\left(h_{1} a_{31}+h_{2} a_{32}+h_{3} a_{33}\right) \omega \mathrm{d} \omega+\text { constant }
\end{aligned}
$$

Putting the values (5) in terms of $\bar{p}, \bar{q}, \bar{r}$, the integrals (16) reduces to

$$
\begin{aligned}
& \frac{1}{2}\left(A \bar{p}^{2}+B \bar{q}^{2}+C \bar{r}^{2}\right)+\frac{3 \omega^{2}}{2}\left(A a_{31}^{2}+B a_{32}^{2}+C a_{33}^{2}\right) \\
& -\frac{\omega^{2}}{2}\left(A a_{21}^{2}+B a_{22}^{2}+C a_{33}^{2}\right)-\omega^{2}\left(h_{1} a_{31}+h_{2} a_{32}+h_{3} a_{33}\right) \\
& =\int\left\{A a_{21}\left(\bar{p}+\omega a_{21}\right)+B a_{22}\left(\bar{q}+\omega a_{22}\right)+C a_{23}\left(\bar{r}+\omega a_{23}\right)\right\} \mathrm{d} \omega \\
& \quad+3 \int\left(A a_{31}^{2}+B a_{32}^{2}+C a_{33}^{2}\right) \omega \mathrm{d} \omega-2 \int \omega\left(h_{1} a_{31}+h_{2} a_{32}+h_{3} a_{33}\right) \mathrm{d} \omega+\text { constant }
\end{aligned}
$$

\section{0rientation of Equilibria}

For the equilibrium positions, we shall put $\alpha=\alpha_{0}=$ constant, $\beta=\beta_{0}=$ constant , $\gamma=\gamma_{0}=$ constant in the Equations (2)-(4) and whence we have

$$
\begin{aligned}
& (C-B)\left(a_{22} a_{23}-3 a_{32} a_{33}\right)-h_{2} a_{13}+h_{3} a_{12}=0 \\
& (A-C)\left(a_{23} a_{21}-3 a_{33} a_{31}\right)-h_{3} a_{11}+h_{1} a_{13}=0 \\
& (B-A)\left(a_{21} a_{22}-3 a_{31} a_{32}\right)-h_{1} a_{12}+h_{2} a_{11}=0
\end{aligned}
$$

These equations are entirely identical with those found by [5] and so to avoid repetition of the procedure we shall only summarize the results obtained in the referral papers [1]-[3] obtained for different case, i.e., for

(a) $h_{1} \neq 0, h_{2}=0=h_{3}$

(b) $h_{1}, h_{3} \neq 0, h_{2}=0$

(c) $h_{1}, h_{2}$ and $h_{3}$ are all non- zero.

In case (a) there are at most 24 solutions depending on the parameters $(x, y)$ introduced in the referral paper and the least number is eight. In case (b) which has been studied in paper [2], three groups of solution are found which we shall not mention and in case (c) the solution involves a complicated equation of degree not less than twelve and it has not been solved. Thus we find that the orientation of equilibrium points does not change by taking an elliptic orbit of a satellite instead of a circular one.

\section{Sufficient Condition for Stability}

To investigate the sufficient condition of stability of equilibrium orientation of the problem, we shall consider the energy integral as in the referral papers of Sarychev and others [1]-[3]. For this we shall take

$\alpha=\alpha_{0}+\bar{\alpha}, \beta=\beta_{0}+\bar{\beta}$ and $\gamma=\gamma_{0}+\bar{\gamma}$, where $\left(\alpha_{0}, \beta_{0}, \gamma_{0}\right)$ is an equilibrium point and $((\bar{\alpha}, \bar{\beta}, \bar{\gamma})$ are small variations to the different of the equilibrium points. Putting these values in the energy integral in the circular case $\left(\omega=\omega_{0}=\right.$ constant $)$. It reduces to

$$
A \bar{p}^{2}+B \bar{q}^{2}+C \bar{r}^{2}+\omega_{0}\left(A_{\alpha \alpha} \bar{\alpha}^{2}+A_{\beta \beta} \bar{\beta}^{2}+A_{\gamma \gamma} \bar{\gamma}^{2}+2 A_{\alpha \beta} \bar{\alpha} \bar{\beta}+2 A_{\alpha \gamma} \overline{\alpha \gamma}+2 A_{\beta \gamma} \bar{\beta} \bar{\gamma}\right)+\sum \text { = constant . }
$$


( $\sum=$ terms of higher order than those of second).

where

$$
\begin{aligned}
& A_{\alpha \alpha}^{l}=3(A-C)\left(\bar{a}_{11}^{2}-\bar{a}_{31}^{2}\right)+3(B-C)\left(\bar{a}_{12}^{2}-\bar{a}_{32}^{2}\right)+K_{\alpha \alpha}^{l} \\
& A_{\beta \beta}^{l}=\left[(B-A)-(B-C) \sin ^{2} \gamma_{0}\right]\left[1+3 \sin ^{2} \alpha_{0}\right] \cos ^{2} \beta_{0} \\
&-\frac{3}{4}(B-C) \sin ^{2} \alpha_{0} \sin \beta_{0} \sin 2 \gamma_{0}+K_{\beta \beta}^{l} \\
& A_{\beta \beta}^{l}=(B-C)\left[\left(\bar{a}_{22}^{2}-\bar{a}_{23}^{2}\right)-3\left(\bar{a}_{32}^{2}-\bar{a}_{33}^{2}\right)\right]+K_{\gamma \gamma}^{l} \\
& A_{\alpha \beta}^{l}=3(B-C)\left[\left(\bar{a}_{32} \cos \alpha_{0}-\bar{a}_{12} \sin \alpha_{0}\right) \bar{a}_{22}-\frac{3}{2}(A-C) \sin 2 \alpha_{0} \sin 2 \beta_{0}\right]+K_{\alpha \beta}^{l} \\
& A_{\gamma \gamma}^{l}=-3(B-C)\left(\bar{a}_{12} \bar{a}_{33}-\bar{a}_{13} \bar{a}_{32}\right)+K_{\alpha \gamma}^{l} \\
& A_{\beta \gamma}^{l}=-\frac{1}{2}(B-C)\left[6\left(\bar{a}_{33} \cos \gamma_{0}-\bar{a}_{32} \sin \gamma_{0}\right) \bar{a}_{31}+\sin 2 \beta_{0} \sin 2 \gamma_{0}\right]+K_{\beta \gamma}^{l}
\end{aligned}
$$

Here

$$
\bar{a}_{i j}=a_{i j}\left(\alpha_{0}, \beta_{0}, \gamma_{0}\right)(i=1,2,3)
$$

$K^{1}$ corresponds to the case when $h_{1} \neq 0, h_{2}=0, h_{3}=0$

$K^{2}$ corresponds to the case when $h_{1}=0, h_{2}=0, h_{3} \neq 0$

$K^{3}$ corresponds to the case when $h_{1} \neq 0, h_{2} \neq 0, h_{3} \neq 0$

$K_{\alpha \alpha}^{1}=h_{1} \bar{a}_{11}, \quad K_{\beta \beta}^{1}=h_{1} \bar{a}_{11}, \quad K_{\gamma /}^{1}=0$

$K_{\alpha \beta}^{1}=-h_{1} \sin \alpha_{0} \sin \beta_{0}$

$K_{\alpha \beta}^{2}=-h_{1} \sin \alpha_{0} \sin \beta_{0}-h_{3} \bar{a}_{31} \sin \gamma_{0}$

$K_{\alpha \beta}^{3}=-h_{1} \sin \alpha_{0} \sin \beta_{0}-h_{2} \bar{a}_{31} \cos \gamma_{0}-h_{3} \bar{a}_{31} \sin \gamma_{0}$

$K_{\beta \beta}^{2}=h_{1} \bar{a}_{11}+h_{3} \bar{a}_{21} \cos \alpha_{0} \sin \gamma_{0}$

$K_{\beta \beta}^{3}=h_{1} \bar{a}_{11}+h_{2}\left(-\cos \alpha_{0} \sin \beta_{0} \cos \gamma_{0}\right)+h_{3} \bar{a}_{21} \cos \alpha_{0} \sin \gamma_{0}$

$K_{\beta \gamma}^{1}=0$

$K_{\beta \gamma}^{2}=-h_{3} \bar{a}_{11} \cos \gamma_{0}$

$K_{\beta \gamma}^{3}=-h_{2} \cos \alpha_{0} \cos \beta \sin \gamma_{0}-h_{3} \bar{a}_{11} \cos \gamma_{0}$

$K_{r \gamma}^{2}=h_{3} \bar{a}_{13}$

$K_{\gamma /}^{3}=h_{2} a_{12}+h_{3} a_{13}$

$K_{\alpha \alpha}^{2}=h_{1} \bar{a}_{11}+h_{3} \bar{a}_{13}$

$K_{\alpha \alpha}^{3}=h_{1} \bar{a}_{11}+h_{2} \bar{a}_{12}+h_{3} \bar{a}_{13}$

$K_{\alpha \gamma}^{1}=0, K_{\alpha \gamma}^{2}=h_{3} \bar{a}_{32}, \quad K_{\alpha \gamma}^{3}=a_{33} h_{2}+h_{3} a_{32}$,

Sufficient conditions for stability have been found for the circular case in the form of showing the energy integral to be positive definite and on the application of Lyapunov's theorem for stability the result follows. The conditions are given in the referred paper [1] [3] and to avoid the repetition we shall not write them here.

Corresponding to the elliptic case we have a non-autonomous system and here also we shall follow the same process. We shall take up the integral (18) and let $V^{N}$ and $Q$ be written for the non-autonomous and quadratic part respectively lying on the right side of the integral (18), i.e, the integral (18) may be written as $V^{N}=Q+$ constant .

1) Firstly, we shall consider the stability condition for a fixed $t=t_{0}$, then since $\omega\left(t_{0}\right)$ is constant, it virtually reduces to the circular case and the sufficient condition for stability will remain the same as for the circu- 
lar case and to avoid repetition we shall only refer to the investigation made by Sarychev and others [1]-[3].

2) Secondly, we shall consider the average value of $\omega$ given by $\omega_{0}=\frac{1}{2 \pi} \int_{0}^{2 \pi} \omega \mathrm{d} t$. Here also we find that $\omega_{0}=\frac{1}{2 \pi} \int_{0}^{2 \pi}(1+e \cos \theta)^{2} \mathrm{~d} t=$ constant . Thus in this case also the problem reduces to the circular case and we shall not proceed further.

3) Here we shall consider the general case of the system being a non-autonomous. Although a positive $V^{N}$ can be found, but as $\frac{\mathrm{d} V}{\mathrm{~d} t}$ is neither zero, nor negative, so to us it appears that by choosing a suitable positive definite integral, the stability cannot be discussed and we need the employment of some other method. Lyapunov's theorem needs a positive definite quadratic function $V^{N}$ and $\frac{\mathrm{d} V^{N}}{\mathrm{~d} t}=0$ or negative and thus it is found that Lyapunov's conditions for stability are not satisfied and no conclusion can be made or it may be unstable.

\section{Conclusion}

Here conditions for stability have been studied referring to the work of [2], Sarychev and others [2]-[4]. In different cases, all reduce to the circular case. So far as the elliptic case is concerned, we took up the work different from the studies made earlier, but as the factor of angular velocity cancels out from the equations, the orientations of the equilibrium points reduce to that for the circular case and it does not need any fresh investigation. Coming to the stability condition for the case of elliptic orbit we have not come to any definite conclusions, although a positive definite quadratic function can be made available as in [1]-[3] as for $V^{N}$, but as $\frac{\mathrm{d} V}{\mathrm{~d} t}$ is neither zero nor negative, so nothing can be concluded except that the motion will be unstable. Only a numerical check up can only determine the definite nature of the equilibrium position. The effect of aerodynamic and gravitational torques plays an important role. The result will be of far importance for the determination of the motion of altitude.

\section{References}

[1] Sarychev, V.A. (1965) Dynamics of a Satellite Gravitational Stabilization with Consideration of Atmospheric Resistance. Proceedings of the Eleventh International Congress of Applied Mechanics Munich (Germany), 1964, 429-435.

[2] Beletskii, V.V. (1966) Motion of an Artificial Satellite about Its Centre of Mass. NASA TTF-429.

[3] DeBra, D.B. (1959) The Effect of Aerodynamic Forces on Satellite Attitude. Journal of the Astronautical Sciences, 6, 40-45.

[4] Frik, M.A. (1970) Attitude Stability of Satellite Subjected to Gravity Gradient and Aerodynamic Torques. AIAA Journal, 8, 1780-1785. http://dx.doi.org/10.2514/3.5990

[5] Sarychev, V.A. and Mirer, S.A. (2000) Relative Equilibria Subjected to Gravitational and Aerodynamic Torques. Celestial Mechanics and Dynamical Astronomy, 76, 55-68. http://dx.doi.org/10.1023/A:1008389730047

[6] Sarychev, V.A., Mirer, S.A, Degtyarev, A.A. and Duarte, E.K. (2007) Investigation of Equilibria of a Satellite Subjected to Gravitational and Aerodynamic Torques. Celestial Mechanics and Dynamical Astronomy, 97, 267-287. http://dx.doi.org/10.1007/s10569-006-9064-3

[7] Sarychev, V.A., Mirer, S.A. and Degtyarev, A.A. (2008) Equilibria of a Satellite Subjected to Gravitational and Aerodynamic Torques with Pressure Centre in a Principal Plane of Inertia. Celestial Mechanics and Dynamical Astronomy, 100, 301-318. http://dx.doi.org/10.1007/s10569-008-9126-9 